\title{
Development of a system for automated control of oil transportation in the Arctic region to prevent the formation of paraffin deposits in pipelines
}

\author{
Alexandra Kopteva ${ }^{1 *}$, Vladimir Koptev ${ }^{1}$, Vadim Malarev ${ }^{1}$, and Taisiya Ushkova ${ }^{1}$ \\ ${ }^{1}$ Saint-Petersburg Mining University, St. Petersburg, Russian Federation
}

\begin{abstract}
The paper introduces a unique methodology of preventing and controlling the formation of paraffin deposits in oil pipelines, which is a matter of urgency in regions with negative temperatures, including the Arctic regions. The paper presents the configuration and specific parameters of the developed measuring unit. The proposed measuring unit is capable of detecting the formation of a paraffin layer during its early stages and predicting its composition. Simultaneous use of two radioisotope sensors allows increasing the performance capabilities of the measuring unit, namely to detect the formation of a paraffin layer in the early stages and to issue a signal to amplify the control signal to control the oil pipeline paraffinisation. In addition, the use of a radioisotope unit instead of a single sensor will increase the accuracy of component-wise flow rate measurement, by making an allowance for paraffin deposits formation. The use of customized information processing algorithms also allows determining oil viscosity with high accuracy. The paper deals with an optimization of a system for automated control of a promising method of electric heating via the skin-cables, wherein safety factors of up to $36 \%$ are currently used, i.e. in most cases, $1 / 3$ excess electric power is generated. The safety of using the radioisotope measuring technique is proven.
\end{abstract}

\section{Introduction}

Pipeline transport holds a leading position among other types of oil transportation, owing to many of its advantages and ensures the transportation of about $90 \%$ of the total amount of oil produced in Russia. As to the Arctic region, pipeline transport is not yet the main one, which, to a greater extent, is caused by high power consumption associated with lowering the viscosity of produced oil and controlling the formation of paraffin deposits [1-3]. This paper introduces a method of increasing the energy efficiency of arctic oil transportation through pipelines and preventing the formation of a paraffin layer.

The proposed technique for controlling the formation of paraffin deposits during oil transportation is relevant for use in all regions, producing viscous oil, especially under conditions of the Arctic region. Due to specific features of oil produced in the Arctic region (heavy, highly viscous oil), it is apparent that the use of noncontact systems for controlling the said process is required. Among all non-contact control methods being developed according to the existing state of art, it is the radioisotope method only that has shown the efficiency when used with heterogeneous streams, which include oil flows. In addition, under conditions of the Arctic region, heated oil is usually transported at very low ambient temperatures (up to - 55 degrees), which creates a large temperature gradient and significantly increases the risk of occurring a paraffin layer on the inner walls of oil pipelines, which in combination with the increased complexity of performing repair and refining operations, makes critical the use of equipment for the paraffin layer detection [4-5]. According to the results of the studies, it was revealed that only the radioisotope control method, among all existing ones, is able to detect the formation of the paraffin layer in the early stages and therefore the only possible way to solve the assigned task is to create automated systems for controlling the formation of paraffin deposits based upon this method [6].

One of the most promising means for controlling viscosity and preventing the formation of paraffin deposits is electric heating via the skin-cables; moreover, one of the companies-leaders in the production of this technology is a Russian company [7]. The skin-system principle of operation is based on the use of special heating elements using the skin-effect phenomenon and the proximity effect in conductors of ferromagnetic materials operated with an alternating current of industrial frequency [8-10]. The heating element is a tube of low-carbon steel, inside which a conductor of non-magnetic material is placed. The conductor, at the end of the heating arm, is securely connected to the steel tube, and an alternating voltage is supplied between the tube and the conductor at the beginning of the arm. Owing to the skin-effect, the current flows along the

\footnotetext{
* Corresponding author: alexandrakopteva@gmail.com
} 


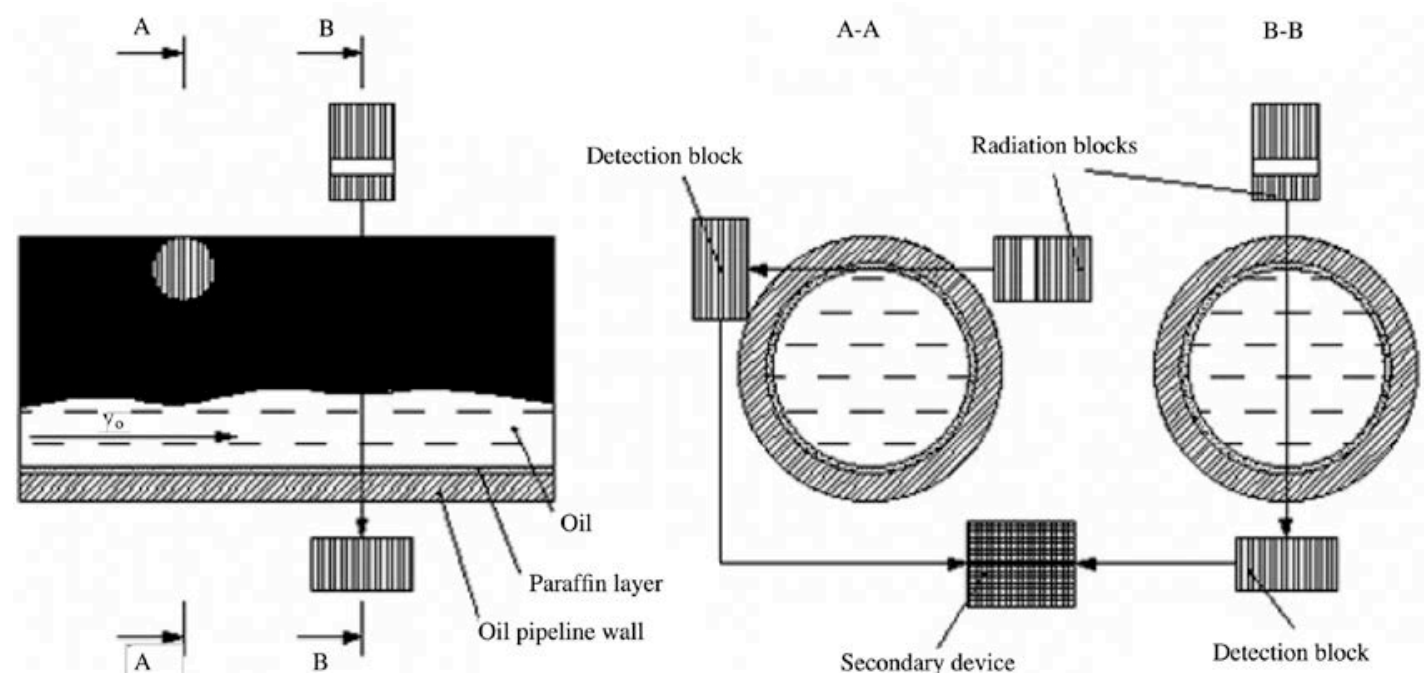

Fig. 1. Configuration of a radioisotope measuring unit for detecting the formation of paraffin deposits.

inner surface of the skin-heater tube, while it is substantially missing on its outer surface [11-13]. The absence of electric potentials makes the system safe for operating personnel. When the current flows, heat is released in both conductors $(60-80 \%$ of the heat is generated in a steel tube and only $20-40 \%$ in a conductor with a copper core).

\section{Methods}

In the general case, the electric system for the oil pipeline heating via the skin-cables consists of a heater, a power supply subsystem, and a control subsystem. The heating skin-cables that serve as the system's control element are used as a heater; the skin-cables are secured directly to the pipeline, i.e. to the controlled object. The control subsystem equipment includes a system of measuring instruments (negative feedback is based on a temperature sensor), analog-to-digital and digital-toanalog converters, a controller that processes the data obtained from the sensors and generates the effects by the power supply subsystem (ACS control element) [14]. The power supply system is implemented in the form of a complete transformer substation, which includes distribution cells of high and low sides, a specialized balance-unbalance transformer [15].

Currently, the control action by voltage in the skincables is being generated based on the temperature sensors readings. Moreover, it is worth noting that in addition to temperature, the paraffinisation process is affected by pressure in the pipeline, concentration of high molecular weight hydrocarbons of paraffin series, the difference in temperature of ambient medium and oil flow, the amount of free gas in oil flow, the flow velocity, water cut, and component-wise oil composition (in particular percentage of light fractions), oil density, at the initial stages of paraffinisation - the properties of the pipeline surface, and its thickness after the formation of a paraffin layer. Due to the absence of a direct relationship between the temperature sensor readings and the process of paraffinisation, safety factors of up to $36 \%$ are used when generating the control action, which makes this method of reducing oil viscosity and controlling the formation of paraffin deposits less costeffective and results to an increase in the prime cost of oil and petroleum products as a whole [15].

To optimize the use of electric heating, it is necessary to apply a new measuring unit, capable to detect the formation of a paraffin layer at an early stage, as a part of the automated control system. The developed measuring unit uses ionizing radiation technology, which was chosen on the basis of comparative analysis [6]. The schematic diagram of the measuring unit is presented in fig. 1.

The measurements, carried out via each radioisotope sensor, are based on the principle of ionizing radiation attenuation by the oil stream, flowing between the radiation and the detection blocks. Ionizing radiation occurs due to the decay of the $\mathrm{Cs}_{137}$ radionuclide with the release of photons with an average energy of 661 $\mathrm{keV}$. The emitted photons, when passing through a narrow hole in the radiation block, pass through a pipeline with the oil stream, being subjected to photoelectric absorption and the effects of Compton scattering. As a result, the detection block registers the flux of gamma rays, with a lower average energy, which can be calculated based on the known initial radiation intensity and the measured attenuated intensity $I$ according to the Lambert-Beer law [6]:

$$
I=I_{0} \exp (-\mu \cdot \rho \cdot d),
$$

where $\rho$ - is the density of the controlled medium; $\mu$ - is the mass attenuation coefficient of radiation by the medium; $\mathrm{d}$ - is the linear size of the controlled medium.

The combined determination of ionizing radiation attenuation of velocities of the liquid and gas components of the oil flow and the density of the transported oil by the radioisotope sensor is already effectively used in the oil industry to determine component-wise mass flow rate $[6,16]$. Simultaneous use of two radioisotope sensors allows increasing the 


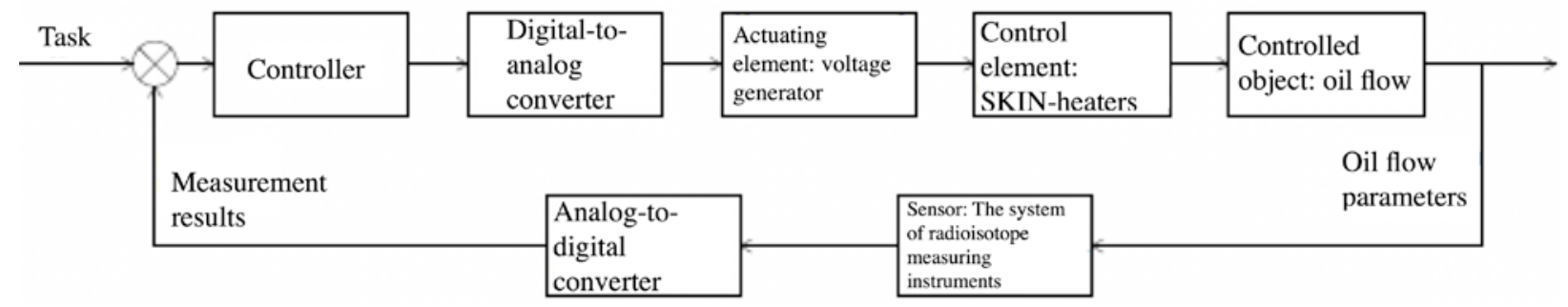

Fig. 2. Schematic diagram of the developed system for preventing the formation of paraffin deposits.

performance capabilities of the measuring unit, namely to detect the formation of a paraffin layer in the early stages and to issue a signal to amplify the control signal to control the oil pipeline paraffinisation. In addition, the use of a radioisotope unit instead of a single sensor will increase the accuracy of component-wise flow rate measurement, by making an allowance for paraffin deposits formation.

To detect paraffinisation process, two radioisotope sensors should rest on the pipeline surface according to the developed configuration. The radiation and detection blocks of the first sensor are arranged coaxially, so that a narrow flux of gamma rays passes near the pipeline wall and controls the most risky point in terms of paraffinisation both along the pipeline's length and cross-section. The most risky point in terms of paraffinisation is determined based on the experience of work at oil pipelines, and when commissioning new oil pipelines or using new means of controlling paraffinisation process, based on the results of mathematical modeling. The radiation and detection blocks of the second sensor are also arranged coaxially, being always in a vertical plane that lies in the axis of the pipeline. The distance between the two sensors of the device should be minimal, to achieve measurement accuracy and sufficient for convenient mounting and operation, and is set to $20 \mathrm{~cm}$.

Narrow gamma-ray beams of the developed radioisotope unit are invariant relative to each other, since the photons that constitute them are neutral particles being unable to interact. Rare cases of the interaction of two photons were recorded during the decay of a photon into an electron and a positron and the interaction of a second photon with one of the elementary particles, but such a decay is possible only at photon energy exceeding tens of $\mathrm{MeV}$, therefore it is not a case for the developed measuring unit, using radionuclide $\mathrm{Cs}_{137}$ with photon energy of $0.662 \mathrm{MeV}$. However, part of energy from narrow gamma-ray beams is transmitted to the controlled oil flow, and under the sensors' misconfiguration, this energy can reduce the pipeline paraffinisation at the unit's place of location, affecting the accuracy of measurements taken along the pipeline length. To eliminate the impact of measurements made by the measuring unit on paraffinisation at the detection point, it is important to install the first radioisotope sensor that controls the paraffin layer near the pipeline wall, upstream of the oil flow in the pipeline, compared to the second one. Thus, with the described configuration of two radioisotope sensors taken into account, their combined use does not affect the measurements performed by the sensors separately.

The formation of paraffin deposits is directly detected in the secondary device of the radioisotope measuring unit, which is a controller with a built-in data processing algorithm [17-18]. Two arrays of data on measured intensity come to the controller input: the first data array comes from the first sensor and contains information on the density of the controlled substance in the most risky point of the pipeline in terms of paraffinisation, the second array, correspondingly, comes from the second sensor and characterizes the density of the flow transported through the pipeline, taken as a whole. Firstly, the controller processes each data array separately, making them invariant to the intensity of radiation, scattered in the pipeline wall and to fluctuations in the intensity caused by the presence of the gas component in the oil flow, as well as determining flow velocities of the liquid and gas component of the oil-gas-water flow in the pipeline using a marker method. Then, based on the known distance between the two sensors and the calculated velocity of the liquid component of the oil flow, the controller brings the data of the two arrays to the same cross-section of the oil flow. Further, the controller compares the said data arrays by the mass attenuation coefficient of radiation by the controlled medium and, if the difference exceeds twice the maximum value of the absolute error, caused by the non-absolute constancy of the primary radiation intensities of the radionuclides, the controller issues a signal about the presence of a paraffin layer on the pipeline wall. The algorithm of the developed system is presented in figure 2.

\section{Results}

Upon the receipt of the input data $\rho_{\mathrm{o}}[\mathrm{n}]$ (average oil density), $\rho_{\text {os }}[n]$ (oil density at the pipe wall), $v_{\mathrm{gs}}[\mathrm{n}]$ (gas velocity at the pipe wall), $v_{\text {os }}[n]$ (liquid velocity at the pipe wall), the controller checks their reliability, i.e. compares the obtained values with the values processed during the previous cycle and determines the possibility of such a change for the sampling interval. The check is carried out based on the following formulas: 


$$
\begin{aligned}
& \left|\rho_{O}[n]-\rho_{O}[n-1]\right|>\rho^{*} ; \\
& \left|v_{l s}[n]-v_{l s}[n-1]\right|>v_{l s}^{*} ;, \\
& \left|v_{g s}[n]-v_{g s}[n-1]\right|>v_{g s}^{*} ; \\
& \left|\rho_{O s}[n]-\rho_{O s}[n-1]\right|>\rho^{*} .
\end{aligned}
$$

The failed check witnesses in favor of a malfunction of measuring instruments, processing means, data received therefrom or an emergency situation on the oil pipeline and results to sending a failure signal to higher levels of the automated control system and maintaining the control action (voltage on the skin-conductors) at the level, generated during the previous control cycle prior to the receipt of control signals from higher levels of the automated control system.

If the check of the input data was successful based on these data and the data obtained during previous cycles of the algorithm operation, the beginning of the formation of paraffin deposits on the walls of the detected oil pipeline is detected. The determination is carried out based on the following formulas:

$$
\begin{aligned}
& \left|\rho_{O s}[n]-\rho_{O}[n-1]\right|>D_{\rho}^{* *} ; \\
& R_{\rho_{0 s}}(\tau)=\lim \frac{1}{T} \int_{0}^{T} \rho_{O s}(t) \cdot \rho_{O s}(t+\tau) d t=\text { const } \pm \Delta_{\rho} ; \\
& R_{v g s}(\tau)=\lim \frac{1}{T} \int_{0}^{T} v_{g s}(t) \cdot v_{g s}(t+\tau) d t=\text { const } \pm \Delta_{v g} ; \\
& R_{v l s}(\tau)=\lim \frac{1}{T} \int_{0}^{T} v_{l s}(t) \cdot v_{l s}(t+\tau) d t=\text { const } \pm \Delta_{v l} .
\end{aligned}
$$

In addition to checking the evidence of paraffin deposits formation at this stage of performing calculations, the controller verifies the expiration of the waiting time for the paraffin layer response to the last change in the control action:

$$
R_{U}(\tau)=\lim \frac{1}{T_{p}} \int_{0}^{T_{p}} \dot{U}(t) \dot{U}(t+\tau) \mathrm{dt}=\text { const. }
$$

If the performed calculations witness in favor of the formation of a paraffin layer and of the expiration of the waiting time for the paraffin layer response to the last change in the control action, then it obviously results to the need to increase the temperature of heating, via the skin-conductors, the transported oil flow above the temperature of paraffin deposition, i.e. the need to increase the voltage on the skin-conductors [19]. The controller calculates the control action, raising the voltage higher than it was during the previous control cycle by the minimum step, permissible under the technical conditions of controlling $\mathrm{u}_{\min }$ :

$$
\mathrm{U}[\mathrm{n}]=\mathrm{U}[\mathrm{n}-1]+\mathrm{u}_{\min } .
$$

The calculated value of the control action is checked for compliance with the range of values of the minimum and maximum possible control action $\mathrm{U}_{\text {min }}<\mathrm{U}[\mathrm{n}]<\mathrm{U}_{\text {max }}$, depending on the characteristics of the skin-cables and specific operating conditions. The calculated voltage value, exceeding the maximum one, witnesses in favor of the inability of this means of controlling the formation of paraffin deposits to perform the transportation of this type of oil under specified operating conditions, as well as calls for the need to use other means and provides the maximum possible control action on the skin-conductors. If the calculated value falls within the voltage range that is technically feasible for the specified operating conditions, then comes its output to the device generating voltage on the skinconductors.

If, the calculations, performed at the second stage of verification, indicate the presence of a paraffin layer, and witness in favor of the fact that the waiting time for the paraffin layer response to the last change in the control action has not yet expired, then the controller outputs the control action of the previous cycle:

$$
\mathrm{U}[\mathrm{n}]=\mathrm{U}[\mathrm{n}-1] .
$$

If the calculations, performed at the second stage of verification, confirm the absence of a paraffin layer, and witness in favor of the fact that the waiting time for the response of the paraffin layer to the last change in the control action has expired, the controller, in order to reduce the power consumption for controlling paraffinisation, reduces the control action on the voltage generator on the skin-conductors by the minimum step permissible under the technical conditions of controlling $u_{\min }$, but not allowing the voltage drop below the minimum [20]:

$$
\mathrm{U}[\mathrm{n}]=\mathrm{U}[\mathrm{n}-1]-\mathrm{u}_{\min } \geq U_{\min } .
$$

In addition to the basic algorithm for determining paraffinisation, the protection algorithms against false positive must be provided in the controller of a radioisotope measuring unit. False positives of the system can be caused by free solid particles formed in the flow, for example, asphalt-resin-paraffin conglomerates or corrosion products of the pipeline walls, which do not accumulate on the pipeline walls, but move freely in the flow and do not cause negative effect typical for the paraffin layer. To prevent such false positives occurrence, the controller checks the constancy of the fulfillment of the condition of paraffinisation for a certain period of time and only after this is done outputs a signal about the oil pipeline paraffinisation to external devices. The time period is determined by the ratio of the linear size of the maximum solid particle detected in the pipeline, for most pipelines it makes $12.5 \mathrm{~mm}$, to the calculated velocity of the oil flow liquid component. For example, for a flow velocity of $0.5 \mathrm{~m} / \mathrm{s}$, the time period will be $0.025 \mathrm{~s}$, and the number of checks, required for an accurate signal about paraffinisation at a measurement frequency of $250 \mathrm{~Hz}$, will be 7 . In most cases, the flow velocity is greater, and, as a result, the number of checks is less than 7 , and the speed of the measuring unit response is more than $0.025 \mathrm{~s}$.

In addition, the controller provides for the displacement of a narrow gamma-ray beam from a controlled point or the ingress of other bodies into the 


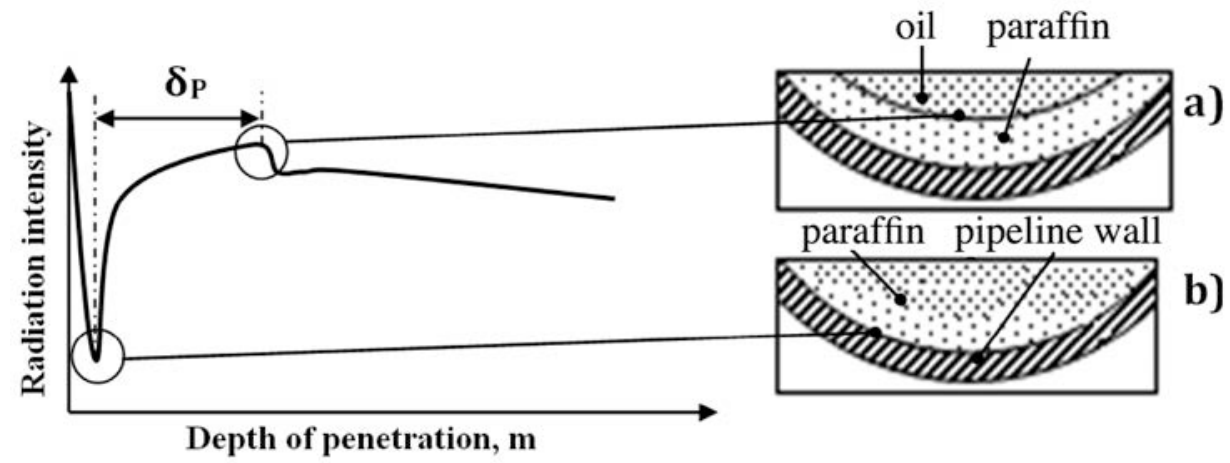

Fig. 3. Schematic diagram of the developed system for preventing the formation of paraffin deposits.

controlled zone, limiting the range of possible values of intensity attenuation from the values recorded under an empty oil pipeline to the values typical for the said oil pipeline, provided that the densest paraffin deposits are present, and issues a signal about error when measurements fall outside the specified range. Thus, the developed measuring unit meets the requirements of modern metrology, owing to eliminating the impact of unforeseen circumstances on the measurement results.

Experimental studies performed on the basis of "Complex-Resource", LLC proved the difference between the densities of the paraffin layer and the oil flowing through the pipeline and the ability of the radioisotope sensor to detect the pipeline paraffinisation. The experiment consisted in moving a radioisotope sensor at a predetermined speed along the vertical axis of the pipeline with a known thickness of paraffin deposits and recording the time of occurrence of jumps in the measured intensity that are typical for a change in the controlled medium. The obtained diagram of changes in the measured intensity depending on the depth of penetration and the cross-sectional point of the pipeline, for which jumps are typical, are presented in fig.3 [6, $16]$.

The conducted experiment revealed the accuracy of determining the thickness of a paraffin layer of $0.5 \mathrm{~mm}$. The radioisotope measuring unit, described in this paper, is static and, as a result, has even greater accuracy and reliability.

The safety certification of radioisotope sensors, based on which the developed measuring unit has been implemented, was also made. The certification showed the maximal recorded dose rate of equivalent radiation at a distance of $1 \mathrm{~m}$ equal to $0.1 \mu \mathrm{Sv} / \mathrm{h}$, which is 200 times less than is established for measuring instruments by the sanitary rules of protection against radiation [21]. Since the radioisotope radiation does not have mutual amplification, but merely is added, the maximum recorded dose rate of equivalent radiation at a distance of $1 \mathrm{~m}$ from the developed measuring unit will not exceed $0.2 \mu \mathrm{Sv} / \mathrm{h}$, which is 100 times less than is established by norms and standards. In addition, the operation of the radioisotope measuring unit is completely automated and does not require the presence of operating personnel, the unit itself is placed in a protective housing, having signs of radiation danger on its surface, and the access is possible only with a special key.

Proceeding from the above, it can be concluded that the measuring system does not harm the health of the enterprise personnel, subject to observing the safety precautions [21].

\section{Discussion}

Thus, this paper describes the principle of operation, components and configuration of a radioisotope measuring unit, capable to implement fully automated, non-contact and continuous detection of a paraffin layer on any static equipment, being in contact with oil. It is on the basis of this measuring unit that makes possible to create automated control systems for preventing, reducing and eliminating the formation of paraffin deposits.

This paper discusses the possibility of automation of oil transportation modes to prevent the formation of paraffin deposits by the example of a new automated control system for controlling the skin-cables. The choice of this method is grounded by the immense prospects for application, ease of automation and a significant increase in its performance when creating an automated control system based on the developed measuring unit.

However, as was already mentioned, under conditions of ever-changing factors and insufficient information on the parameters of a paraffin layer in the skin-cable system, safety factors of up to $36 \%$ are used, i.e., in most cases, electric power is generated by $1 / 3$ times more than necessary, which makes this method of oil transportation less cost-effective, power-consuming, resulting to a decrease in the efficiency of applying electric heating as a whole [22].

The proposed measuring unit is capable of detecting the formation of a paraffin layer in the early stages and predicting its composition. The use of customized information processing algorithms also allows determining oil viscosity with high accuracy. The said performance capabilities of the measuring system create significant prospects for the development of many control systems [22-25].

The introduction of a radioisotope measuring unit into the automated control system for electric heating of oil equipment will not only significantly increase the energy 
efficiency of highly viscous oil transportation, resulting to the reduction of economic losses, but also increase its reliability, prolong the lifespan of oil equipment and reduce the number of emergency oil spills during transportation [6,13,16, 26].

\section{References}

1. L.V. Eder: Oil industry of the Siberian Federal District at the stage of changing the development paradigm. Institutional transformation of the economy: space and time: V International sci. conf.: Collection of reports. 2 (2017)

2. I. V. Filimonova: Structural changes in the oil production of Russia. Ecological Bulletin of Russia. 1 (2018)

3. P.V. Pokhodyaev: Design of a trunk pipeline in the Far North: dis. Siberian Federal University (2017)

4. A. A. Shatokhina, M. A. Misyun: Modeling of paraffin formation processes and transport features of high-viscosity oils. Probl. of Geology and Subsoil Develop.: Proceedings of the XXI Int. Academician named after MA Usov students and young scientists dedicated to the 130th anniversary of the prof. MI Kuchina. 2 (2017)

5. A. Sousa, H. Matos, M. Pereira: Modelling Paraffin Wax Deposition Using Aspen HYSYS and MATLAB. 29th European Symposium on Computer Aided Process Engineering (2019) DOI: 10.1016/B978-0-12-818634-3.50163-6.

6. A. V. Kopteva, V. Y. Koptev: Automatic Measuring System for Oil Stream Paraffin Deposits Parameters. IOP Conf. Ser.: Materials Sci. and Engin. 327, 4 (2018) DOI:10.1088/1757-899X/327/4/042053

7. M.L. Strupinsky, N.N. Khrenkov, A.B. Kuvaldin., Design and operation of electric heating systems in the oil and gas industry: a reference book. - M .: Infra-Engineering (2015)

8. V.R. Chupin, I. Yu. Shelekhov, V.V. Pozhidaev: Industrial electric heating systems. Role and place of information technologies in modern science (2017)

9. V.Frolov, D. Ivanov, E. Smirnova, B. Yushin: Induction hardening of air-plasma coatings. 18th Symp. Phys. Switch. Arc, FSO (2009)

10. V. Frolov, A. Kvashnin: Nonstationary mathematical model of a magnetic arc blast system. Conf.: 2018 IEEE Conf. of Russ. Young Research. in Electr.and Electron. Engin. (EIConRus) (2018) DOI: 10.1109/EIConRus.2018.8317173

11. I.I. Khasanov, R.A. Shakirov, A.Yu. Leontiev, O.Yu. Poletaeva: Review of modern methods of influencing the rheological properties of heavy high-viscosity oils. NefteGazokhimiya. 3 (2018)

12. A. A. Fedin: The use of induction heating of technological pipelines. BBK 1 A28 (2018)

13. A. Belsky, V. Dobush: Oil wellbore electrical heating station with wind-driven power unit. The 10th Int. conf. Electric Power Quality and Supply Reliability (PQ-2016) (2016) DOI: 10.1109/PQ.2016.7724120

14. V.Yu. Bazhin, V.B. Kuskov, Ya.V. Kuskova: Problems of using unclaimed coal and other carbon-containing materials as energy briquettes. Ugol 4 (2019) http://dx.doi.org/10.18796/0041-5790-2019-4-50-54

15. Yu.L. Gukovskiy, Yu.A. Sychev, D.N. Pelenev: The automatic correction of selective action of relay protection system against single phase earth faults in electrical networks of mining enterprises. International Journal of Applied Engineering Research, Research India Publications. 12 (2017)

16. V. Koptev, A. Kopteva, D. Poddubniy: Increase in Energy Efficiency of Oil and Gas Companies by Perfecting of Management Systems. IEEE Conf. of Russ. Young Research. in Electr. and Electron. Engin. (EIConRus) (2019) DOI: 10.1109/EIConRus.2019.8657041.
17. V.A. Shpenst: Complexation of telecommunications and electrical systems in mines and underground facilities. Zapiski Gornogo instituta / Journal of Mining Institute. 235 (2019) DOI: 10.31897/PMI.2019.1.78

18. A. Toropchin, V. Frolov, A. Pipa, D. Uhrlandt: Calculation of the Heat Affected Zone Coupled with the Arc Simulation in Tig Welding Process Considering the Marangoni Effect. Plasma Phys. Techn. 2 (2015)

19. Ding li Zhang, Min Lin: A Deep-Sea Pipeline Skin Effect Electric Heat Tracing System. Energies. 12. 2466 (2019) Doi: $10.3390 /$ en12132466

20. A. Toropchin, V. Frolov, A. Pipa, R. Kozakov, D. Uhrlandt: Influence of the arc plasma parameters on the weld pool profile in TIG welding. J. of Physics Conf. Ser. 550 (2014) DOI: 10.1088/1742-6596/550/1/012004

21. J. Hoffmann et al.: Safety testing and operational procedures for self-developed radiofrequency coils. NMR in Biomedicine. 29, 9 (2016)

22. A. N. Skamyin, M. S. Kovalchuk: Energy efficiency improving of reactive power compensation devices. IEEE Conf. of Russ. Young Research. in Electr. and Electron. Engin. (EIConRus) (2018) DOI: 10.1109/EIConRus.2018.8317207

23. V.Yu. Bazhin, V.B. Kuskov, Ya.V. Kuskova: Processing of Low-Demand Coal and Other CarbonContaining Materials for Energy Production Purposes. Inzynieria Mineralna (Mineral Engineering) 1, 43 (2019) http://doi.org/10.29227/IM-2019-01-37

24. D.Z. Valiev, R.A. Kemalov, A.F. Kemalov: Regulating temperature of oil saturation with paraffins to avoid asphaltene, resin and paraffin substances deposition during oil production. IOP Conf. Seri.: Earth and Environmental Science. 282 (2019) Doi: 10.1088/1755-1315/282/1/012023

25. A.A. Veprikov, B.N. Abramovich: Prospects for IGBT application in electrical power converters for industrial highcurrent DC loads. IOP Conf. Ser. Materials Science and Engineering. $\quad \mathbf{5 6 0}$ (2019) DOI: 10.1088/1757899X/560/1/012131

26. I.M. Zaidullin, G.R. Valieva, A.Kh. Aliev, A.I. Lakhova, A. Vakhin, S. Petrov: Influence of the Structure of Heavy Oil Disperse System on its Rheological Properties Under Steam-Heat Treatment Conditions. Chemistry and Technology of Fuels and Oils. 53, 4 (2017) Doi: 10.1007/s10553-017-0825-3 